\title{
Differential Cooperative Communications with Space-Time Network Coding
}

\author{
Zhenzhen $\mathrm{Gao}^{* \dagger}$,Hung-Quoc Lai* ${ }^{*}$, and K. J. Ray Liu* \\ *Department of Electrical and Computer Engineering, University of Maryland, College Park, MD 20742, USA \\ Email:\{ygggzhen, kjrliu\}@umd.edu \\ ${ }^{\dagger}$ Department of Information and Communication Engineering, Xi' an Jiaotong University, Xi' an, P. R. China 710049 \\ ${ }^{\ddagger}$ US Army RDECOM CERDEC, RDER-STW-NA, Bldg. 245, Aberdeen Proving Ground, MD 21005, USA \\ Email: hungquoc.lai@us.army.mil
}

\begin{abstract}
In multinode cooperative communications, simultaneous transmissions from two or more nodes are challenging due to its asynchronous nature. In addition, channel estimation is a costly task due to the amount of training, especially when the number of cooperative users is large. Considering all these practical challenges in multinode cooperative communications, this paper propose a new transmission scheme, namely differential space-time network coding (DSTNC), to overcome the problems of imperfect synchronization and complicated channel estimation. Each user in the network linearly combines the correctly decoded symbols by a network coding vector, which is designed to achieve full diversity without introducing large time delay. The pairwise error probability (PEP) is analyzed and the design criteria of the DSTNC are derived based on the PEP. The proposed DSTNC scheme can be applied to any number of cooperative users. Simulation results are shown to verify the performance of the proposed transmission scheme.
\end{abstract}

\section{INTRODUCTION}

User cooperation is an effective scheme to introduce spatial diversity in wireless networks without the use of co-located multiple antennas. The distributed antennas from the relays form a virtual antenna array to provide spatial diversity. Different cooperative protocols are devised and the outage performances are analyzed in [1].

Most of the existing studies assume perfect synchronization and perfect channel state information (CSI) available at the destination. However, such an assumption is difficult to be met in practice. First, cooperative communication is asynchronous in nature [2]. The cooperative diversity is provided by different antennas in separated terminals, where each terminal has its own location oscillator (LO). These LO's generate transmit frequencies with certain variations, and that is hard for the destination to estimate and compensate all the frequency offsets at once. Cooperative communication also faces the issue of timing synchronization. It is challenging for the destination to receive all relaying signals simultaneously due to different propagation time, processing time, and timing estimation errors. Second, channel estimation is challenging and costly, and the amount of overhead becomes substantial in multinode wireless systems because the amount of training or convergence time (incurred by blind techniques) grows with the number of links [3]. Therefore, system design that does not require the knowledge of CSI at the destination is a practical consideration [4]. Recently, the coding methods to achieve the cooperative diversity where timing synchronization is not required are discussed in [2], [5], and [6]. Time Division Multiple Access (TDMA) is used in [7], [8] to overcome the imperfect synchronization issue that prohibits two or more nodes from transmitting at the same time. To reduce the total required time slots in TDMA for cooperative communications, wireless network cocast (WNC) and space-time network coding are proposed [7], [8]. Differential modulation schemes in multinode cooperative wireless networks are analyzed in [4].

In this paper, we propose a new concept of differential space-time network coding (DSTNC) to solve the issues of timing and frequency synchronizations and channel estimation. In a cooperative network of $N$ nodes, single transmissions in TDMA mode require $N^{2}$ time slots to transmit $N$ symbols resulting in large transmission delay. The nodes in our network jointly transmit their information in TDMA mode to reduce the required time slots and the total time slots reduces to $2 N$. Instead of repeating the received symbols alternatively in Phase II, each node transmits a new symbol generated from the received information. The method to combine the received information in this paper is quite different from that in [7] and [8] where CDMA is used. Specifically, a combining function, which is determined by a network coding vector, is designed for each node to reduce the transmission delay while achieving full diversity. The network coding here, which is a set of complex parameters optimized for conventional signal constellations, is also different from the traditional network coding, which linearly combines Galois symbols with coding coefficients picked from a finite field of fixed size [9]. We analyze the PEP performance of the proposed DSTNC scheme and derive the design criteria of the network coding matrix. Simulations are provided to validate the performance of the proposed scheme. It can be seen from the simulations that full diversity can be achieved even though not all of the received symbols can be decoded correctly by the user nodes.

Notations: Upper (lower) case boldface letters stand for matrices (vectors). $\mathrm{T}$ and $\mathrm{H}$ denotes transposition and conjugate transposition, respectively. E stands for expectation. $\mathbf{I}_{N}$ is an $N \times N$ unit matrix. $\mathbf{0}_{N}$ is an $N \times 1$ all-zero vector. $\operatorname{diag}\left[d_{1}, \ldots, d_{N}\right]$ is the $N \times N$ diagonal matrix whose $i$ th diagonal entry is $d_{i}$. 


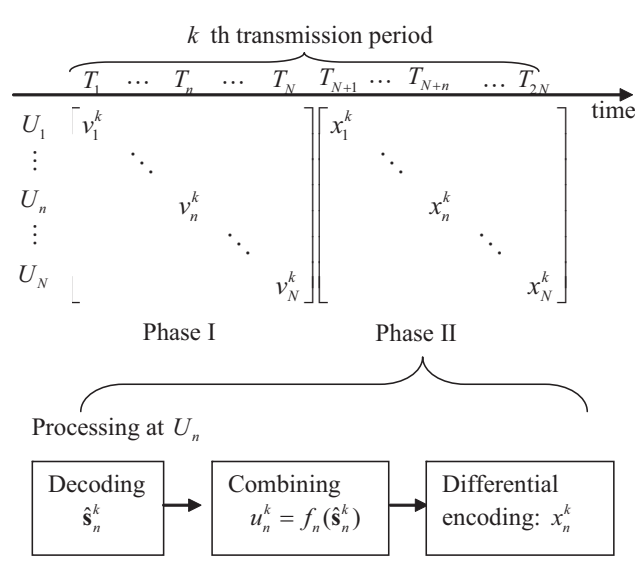

Fig. 1. the $k$ th transmission and processing at the user nodes

\section{SySTEM MOdeL}

Consider a wireless multinode cooperative communication network in which a set of $\mathrm{N}$ user nodes $U_{i}, 1 \leq i \leq N$, cooperate to transmit to the destination node $D$. Each node is equipped with single antenna. Selective decode-and-forward protocol [1] is adopted. For mathematical tractability, we assume that the user nodes can judge whether the received symbols are decoded correctly or not [10]. It is shown in [10] that this assumption is very close to the performance of practical scenario of comparing the received SNR to a threshold, especially when the users operate in high SNR, for example, when the users are close to each other. The channels between any two nodes in the network are modelled as narrowband Rayleigh fading with additive white Gaussian noise (AWGN). Let $g_{m n}$ and $h_{m}$ denote the channel between $U_{m}$ and $U_{n}$ and the channel between $U_{m}$ and $D, \forall m, n \in[1, N]$, respectively. Then $g_{m n} \sim \mathcal{C N}\left(0, \sigma_{m n}^{2}\right)$ and $h_{m} \sim \mathcal{C N}\left(0, \sigma_{m}^{2}\right)$, where the channel variance $\sigma_{m n}^{2} \propto d_{m n}^{-\alpha}, \sigma_{m}^{2} \propto d_{m}^{-\alpha}$ with $d_{m n}$ and $d_{m}$ being the distance between $U_{m}$ and $U_{n}$ and the distance between $U_{m}$ and $D$, respectively and $\alpha$ being the path loss exponent, whose value is usually in range of 2 and 4 .

\section{A. Transmission Model}

In the $k$ th transmission period, the $N$ user nodes need to transmit their information symbols, denoted by a vector $\mathbf{s}^{k}=\left[s_{1}^{k}, s_{2}^{k}, \ldots, s_{N}^{k}\right]^{\mathrm{T}}$, to the common destination $D$ with $k=0$ denoting the initial reference transmission period. $U_{m}$ 's symbol $s_{m}^{k}, m \in[1, N]$, is from an M-QAM constellation $\mathcal{A}$. Each transmission period comprises two phases, the broadcasting phase (Phase I) and the relaying phase (Phase II), each taking place over $N$ time slots. Assuming that frame synchronization has been established, TDMA is used in each phase. Fig. 1 illustrates the signal processing in the $k$ th transmission period. In Phase I, each user node $U_{m}, m \in[1, N]$, is assigned a time slot $T_{m}$ to broadcast its symbol $v_{m}^{k}$ to other nodes $U_{n}(n \neq m)$. Assume that neither $D$ nor the user nodes have any kind of CSI. Thus the transmitted symbol $v_{m}^{k}$ is differentially modulated as $v_{m}^{k}=s_{m}^{k} \frac{v_{m}^{k-1}}{\left|v_{m}^{k-1}\right|}$. The received signal at $U_{n}$ in the $m$ th time slot of Phase I is

$$
y_{m n}^{k}=\sqrt{P_{t}} g_{m n}^{k} v_{m}^{k}+w_{m n}^{k},
$$

where $P_{t}$ is the power constraint of the user nodes, $w_{m n}^{k}$ is zero-mean and $N_{0}$-variance AWGN, $g_{m n}^{k}$ is the channel between $U_{m}$ and $U_{n}$ during the $k t h$ transmission. After decoding, $U_{n}$ obtains a set of estimated symbols from other nodes, i.e., $\hat{\mathbf{s}}_{n}^{k}=\left[\hat{s}_{1 n}^{k}, \ldots ., \hat{s}_{m n}^{k}, \ldots, \hat{s}_{N n}^{k}\right]^{\mathrm{T}}$, where $\hat{s}_{m n}^{k}=\beta_{m n} s_{m}^{k}$ with $\beta_{m n}$ indicating the detection state at $U_{n}$ for $s_{m}^{k}$. $\beta_{m n}$ for $m \neq n$ can be formulated as

$$
\beta_{m n}=\left\{\begin{array}{ll}
1, & \hat{s}_{m n}^{k}=s_{m}^{k} \\
0, & \hat{s}_{m n}^{k} \neq s_{m}^{k}
\end{array} .\right.
$$

It is obvious that $\beta_{n n}=1$ due to the fact that $U_{n}$ always has its own information. The detection states demonstrate that the user nodes just relay the symbols decoded correctly. Then $U_{n}$ combines the elements of the estimation $\hat{\mathbf{s}}_{n}^{k}$ using a linear function to form a unique symbol as follows

$$
u_{n}^{k}=f_{n}\left(\hat{\mathbf{s}}_{n}^{k}\right)=\sum_{m=1}^{N} a_{m n} \hat{s}_{m n}^{k}=\mathbf{a}_{n}^{\mathrm{T}} \hat{\mathbf{s}}_{n}^{k}
$$

with $\mathbf{a}_{n}=\left[a_{1 n}, \ldots, a_{n n}, \ldots, a_{N n}\right]^{\mathrm{T}}$. Assume that $\mathbf{a}_{n}^{\mathrm{T}} \mathbf{a}_{n}=1$. We call $\mathbf{a}_{n}, n \in[1, N]$, as the network coding vector at $U_{n}$.

Because the destination does not have any CSI, the user nodes would transmit the combined symbol $u_{n}^{k}, n=$ $1,2, \ldots, N$, differentially. Each node transmits its symbol in its assigned time slot, and the symbols transmitted in Phase II can be written as a diagonal space-time matrix $\mathbf{D}_{x}^{k}=$ $\operatorname{diag}\left[x_{1}^{k}, \ldots, x_{n}^{k}, \ldots, x_{N}^{k}\right]$, which is generated as follows

$$
\mathbf{D}_{x}^{k}=\mathbf{D}_{u}^{k} \mathbf{D}_{x}^{k-1} \overline{\mathbf{D}}_{x}^{k-1},
$$

where $\mathbf{D}_{x}^{k-1}=\operatorname{diag}\left[x_{1}^{k-1}, \ldots, x_{n}^{k-1}, \ldots, x_{N}^{k-1}\right]$ represents the transmitted space-time block in the $(k-1)$ th transmission period, $\mathbf{D}_{u}^{k}=\operatorname{diag}\left[u_{1}^{k}, \ldots, u_{n}^{k}, \ldots, u_{N}^{k}\right]$, and $\overline{\mathbf{D}}_{x}^{k-1}=$ $\operatorname{diag}\left[\frac{1}{\left|x_{1}^{k-1}\right|}, \ldots, \frac{1}{\left|x_{n}^{k-1}\right|}, \ldots, \frac{1}{\left|x_{N}^{k-1}\right|}\right] . x_{n}^{k}=u_{n}^{k} \frac{x_{n}^{k-1}}{\left|x_{n}^{k-1}\right|}$ is the symbol transmitted from $U_{n}$ in Phase II in the $k$ th period, which is differentially encoded between the current symbol $u_{n}^{k}$ and the transmitted symbol $x_{n}^{k-1}$ in the $(k-1)$ th period. The normalization matrix $\overline{\mathbf{D}}_{x}^{k-1}$ prevents the peak power of the transmit signals from being too large or too small.

At the end of Phase II, the received signals at $D$ of the $k$ th transmission can be written as

$$
\mathbf{y}^{k}=\sqrt{P_{t}} \mathbf{D}_{x}^{k} \mathbf{h}^{k}+\mathbf{w}^{k},
$$

where $\mathbf{y}^{k}=\left[y_{1}^{k}, \ldots, y_{N}^{k}\right]^{\mathrm{T}}, \mathbf{h}^{k}=\left[h_{1}^{k}, \ldots, h_{N}^{k}\right]^{\mathrm{T}}$ denotes the channels between the users and $D$ during the $k$ th transmission, and $\mathbf{w}^{k}=\left[w_{1}^{k}, \ldots, w_{N}^{k}\right]^{\mathrm{T}} \sim \mathcal{C N}\left(\mathbf{0}, \mathbf{I}_{N} N_{0}\right)$ is the received noise vector.

Assuming that the channels do not change significantly over a period of two adjacent transmissions, i.e. $h_{n}^{k}=h_{n}^{k-1}=h_{n}$ and $g_{m n}^{k}=g_{m n}^{k-1}=g_{m n}$, we have $\mathbf{h}^{k}=\mathbf{h}^{k-1}=\mathbf{h}$. Thus the received signals can be expressed as

$$
\begin{aligned}
\mathbf{y}^{k} & =\sqrt{P_{t}} \mathbf{D}_{u}^{k} \overline{\mathbf{D}}_{x}^{k-1} \mathbf{D}_{x}^{k-1} \mathbf{h}+\mathbf{w}^{k} \\
& =\mathbf{D}_{u}^{k} \overline{\mathbf{y}}^{k-1}+\tilde{\mathbf{w}}^{k}
\end{aligned}
$$


where $\overline{\mathbf{y}}^{k-1}=\overline{\mathbf{D}}_{x}^{k-1} \mathbf{y}^{k-1}$ and $\tilde{\mathbf{w}}^{k}=\mathbf{w}^{k}-\mathbf{D}_{u}^{k} \overline{\mathbf{D}}_{x}^{k-1} \mathbf{w}^{k-1}$. The equivalent noise vector $\tilde{\mathbf{w}}^{k}$ is zero-mean with the covariance matrix of

$$
\begin{aligned}
\boldsymbol{\Sigma}_{w} & =\mathrm{E}\left[\tilde{\mathbf{w}}^{k}\left(\tilde{\mathbf{w}}^{k}\right)^{\mathrm{H}}\right] \\
& =\left[\mathbf{I}_{N}+\left(\overline{\mathbf{D}}_{x}^{k-1}\right)^{2} \mathbf{D}_{u}^{k}\left(\mathbf{D}_{u}^{k}\right)^{\mathrm{H}}\right] N_{0}=\boldsymbol{\Lambda}_{w} N_{0},
\end{aligned}
$$

where $\boldsymbol{\Lambda}_{w}=\operatorname{diag}\left[\varepsilon_{1}, \ldots, \varepsilon_{n}, \ldots, \varepsilon_{N}\right]$ and $\varepsilon_{n}=1+\frac{\mathrm{E}\left[\left|u_{n}^{k}\right|^{2}\right]}{\left|x_{n}^{k-1}\right|^{2}}$.

\section{B. Signal Detection}

To make the relationship clear, substitute (2) and (3) into the received signals in (6) to get

$$
\mathbf{y}^{k}=\mathbf{S} \boldsymbol{\Gamma} \mathbf{A} \overline{\mathbf{y}}^{k-1}+\tilde{\mathbf{w}}^{k},
$$

where $\mathbf{S}=\operatorname{diag}\left[\mathbf{s}^{k^{\mathrm{T}}}, \ldots, \mathbf{s}^{k^{\mathrm{T}}}\right], \mathbf{A}=\operatorname{diag}\left[\mathbf{a}_{1}, \ldots, \mathbf{a}_{N}\right]$, and $\boldsymbol{\Gamma}=\operatorname{diag}\left[\beta_{11}, \ldots, \beta_{N 1}, \ldots, \beta_{1 N}, \ldots, \beta_{N N}\right]$.

Since $D$ does not know CSI nor the detection state $\boldsymbol{\Gamma}$, based on the previously received signals, the maximum likelihood detection at $D$ for $\mathbf{s}^{k}$ can be written as

$$
\tilde{\mathbf{s}}^{k}=\arg \min _{\mathbf{s}^{k} \in \mathcal{A}^{N}}\left\{\left[\mathbf{y}^{k}-\mathbf{S A} \overline{\mathbf{y}}^{k-1}\right]^{\mathrm{H}} \boldsymbol{\Sigma}_{w}^{-1}\left[\mathbf{y}^{k}-\mathbf{S} \mathbf{A} \overline{\mathbf{y}}^{k-1}\right]\right\} .
$$

With the assumption that the channels do not change significantly over a period of two adjacent transmissions, the maximum likelihood detection for $s_{m}^{k}$ at $U_{n}$ in Phase I is

$$
\hat{s}_{m n}^{k}=\arg \min _{s_{m} \in \mathcal{A}} \frac{\left|y_{m n}^{k}-s_{m} y_{m n}^{k-1}\right|^{2}}{\xi_{m} N_{0}},
$$

where $\xi_{m}=1+\frac{\mathrm{E}\left[\left|s_{m}^{k}\right|^{2}\right]}{\left|v_{m}^{k-1}\right|^{2}}$.

\section{Performance Analysis And Design of DSTnC}

In this section, the performance of the DSTNC scheme is analyzed, and the PEP is derived. Based on the PEP analysis, we derive two design criteria for the network coding vectors, the diversity criterion and the product criterion.

\section{A. PEP Analysis}

We can well approximate the performance in high SNR situations by using an equivalent coherent receiver model (8) with $\overline{\mathbf{Y}}^{k-1}$ acting as a known channel vector and $\tilde{\mathbf{W}}^{k}$ as the equivalent noise vector. Since $\boldsymbol{\Sigma}_{w}$ is clearly a diagonal matrix, the equivalent noise remains uncorrelated but with unequal variance entries. After normalized by $\boldsymbol{\Sigma}_{w}^{-\frac{1}{2}}$, which can be estimated at $D,(8)$ becomes

$$
\boldsymbol{\Sigma}_{w}^{-\frac{1}{2}} \mathbf{y}^{k}=\boldsymbol{\Sigma}_{w}^{-\frac{1}{2}} \mathbf{S} \boldsymbol{\Gamma} \mathbf{A} \overline{\mathbf{y}}^{k-1}+\overline{\mathbf{w}}^{k},
$$

where $\overline{\mathbf{w}}^{k}=\Sigma_{w}^{-\frac{1}{2}} \tilde{\mathbf{w}}^{k}$ has a covariance matrix $N_{0} \mathbf{I}_{N}$. The normalization just adjusts the signal strength at $D$ so that the noise power is uniform, and it does not affect the codeword error probability.

From the above equation, we can derive the PEP, i.e. the probability of transmitting $\mathbf{s}^{k}$ and deciding in favor of another $\mathbf{c}^{k}=\left[c_{1}^{k}, c_{2}^{k}, \ldots, c_{N}^{k}\right]^{\mathrm{T}}$ at the detector, conditioned by previously received signals and the detection state at the users. The Chernoff bound [11] is given by

$$
P\left(\mathbf{s}^{k} \rightarrow \mathbf{c}^{k} \mid \overline{\mathbf{y}}^{k-1}, \mathcal{S}\right) \leq \exp \left(-\frac{d^{2}(\mathbf{S}, \mathbf{C})}{4 N_{0}}\right),
$$

where $\mathcal{S}=\left\{\beta_{11}, \ldots, \beta_{N 1}, \ldots, \beta_{1 N}, \ldots, \beta_{N N}\right\}$, and $d^{2}(\mathbf{S}, \mathbf{C})$ is the distance between the received signals corresponding to the codeword $\mathbf{s}^{k}$ and $\mathbf{c}^{k}$. The distance can be written as

$$
d^{2}(\mathbf{S}, \mathbf{C})=\left(\Delta \mathbf{S} \boldsymbol{\Gamma} \mathbf{A} \overline{\mathbf{y}}^{k-1}\right)^{\mathrm{H}} \boldsymbol{\Lambda}_{w}^{-1}\left(\Delta \mathbf{S} \boldsymbol{\Gamma} \mathbf{A} \overline{\mathbf{y}}^{k-1}\right),
$$

where $\Delta \mathbf{S}=\mathbf{S}-\mathbf{C}$ with $\mathbf{C}=\operatorname{diag}\left[\mathbf{c}^{k^{\mathrm{T}}}, \ldots, \mathbf{c}^{k^{\mathrm{T}}}\right]$. For moderate or high SNR scenarios, we make the following approximation $\mathbf{y}^{k-1}=\sqrt{P}_{t} \mathbf{D}_{x}^{k-1} \mathbf{h}$. Assuming that channels from different users to $D$ are spatially uncorrelated, averaging the bound in (12) with respect to $h$ results in [11]

$$
\begin{aligned}
P\left(\mathbf{s}^{k} \rightarrow \mathbf{c}^{k} \mid \mathcal{S}\right) & \leq \mathrm{E}_{\mathbf{h}}\left[\exp \left(-\frac{d^{2}(\mathbf{S}, \mathbf{C})}{4 N_{0}}\right)\right] \\
& =\prod_{n=1}^{N}\left(1+\frac{P_{t} \lambda_{n}}{4 N_{0}}\right)^{-1},
\end{aligned}
$$

where $\lambda_{n}$ is the eigenvalue of the following $N \times N$ matrix

$\mathbf{R}_{h}^{\frac{1}{2}}\left(\Delta \mathbf{S} \boldsymbol{\Gamma} \mathbf{A} \overline{\mathbf{D}}_{x}^{k-1} \mathbf{D}_{x}^{k-1}\right)^{\mathrm{H}} \boldsymbol{\Lambda}_{w}^{-1}\left(\Delta \mathbf{S} \boldsymbol{\Gamma} \mathbf{A} \overline{\mathbf{D}}_{x}^{k-1} \mathbf{D}_{x}^{k-1}\right) \mathbf{R}_{h}^{\frac{1}{2}}$

with $\mathbf{R}_{h}=\operatorname{diag}\left[\sigma_{1}^{2}, \ldots, \sigma_{N}^{2}\right]$ being the correlation matrix of $\mathbf{h}$. It is easy to verify that the $n$th eigenvalue is $\lambda_{n}=$ $\frac{\sigma_{n}^{2}}{\varepsilon_{n}}\left|\sum_{m=1}^{N} \beta_{m n} a_{m n} \Delta s_{m}\right|^{2}$ with $\Delta s_{m}=s_{m}^{k}-c_{m}^{k}$.

Averaging with respect to the detection states of the users, the PEP can be written as

$$
P\left(\mathbf{s}^{k} \rightarrow \mathbf{c}^{k}\right) \leq \mathrm{E}_{\beta_{m n}}\left[\prod_{n=1}^{N}\left(1+\frac{P_{t} \lambda_{n}}{4 N_{0}}\right)^{-1}\right] .
$$

Because the signal detection at each user is independent and the detection of symbols from different sources at a certain user is also independent, the order of expectation operator and production operator in (16) can be exchanged.

Assuming that symbol error rate (SER) at $U_{n}$ for the symbols from $U_{m}$ is $p_{m n}, \beta_{m n}$ 's are independent Bernoulli random variables with a distribution

$$
P\left(\beta_{m n}\right)=\left\{\begin{array}{ll}
1-p_{m n}, & \text { for } \beta_{m n}=1 \\
p_{m n}, & \text { for } \beta_{m n}=0
\end{array} .\right.
$$

The SER for M-QAM modulation can be expressed as [12]

$$
p_{m n}=F_{2}\left(1+\frac{b_{q} \gamma_{m n}}{\sin ^{2} \theta}\right)
$$

where $b_{q}=\frac{b_{Q A M}}{2}=\frac{3}{2(M+1)}$ and $\gamma_{m n}$ is the average received SNR at $U_{n}$ for the symbols from $U_{m} \cdot \gamma_{m n}$ can be given as $\gamma_{m n}=\frac{\sigma_{m n}^{2} P_{t}}{\xi_{m} N_{0}}$ where $\xi_{m}$ has been defined in Section II. B. The function $F_{2}(x(\theta))$ is given as [12]

$$
F_{2}(x(\theta))=\frac{4 K}{\pi} \int_{0}^{\frac{\pi}{2}} \frac{1}{x(\theta)} d \theta-\frac{4 K^{2}}{\pi} \int_{0}^{\frac{\pi}{4}} \frac{1}{x(\theta)} d \theta
$$

with $K=1-\frac{1}{\sqrt{M}}$. Let $C_{M}$ equal the following constant

$$
C_{M}=\frac{4 K}{\pi b_{q}} \int_{0}^{\frac{\pi}{2}} \sin ^{2} \theta d \theta-\frac{4 K^{2}}{\pi b_{q}} \int_{0}^{\frac{\pi}{4}} \sin ^{2} \theta d \theta .
$$

Because $1+\frac{b_{q} \gamma_{m n}}{\sin ^{2} \theta} \approx \frac{b_{q} \gamma_{m n}}{\sin ^{2} \theta}$ when SNR is high, $p_{m n}$ can be approximated as $p_{m n}=C_{M} \gamma_{m n}^{-1}$ for high SNR. 
Averaging with respect to all possibilities of the detection state, the PEP can be approximated as

$$
\begin{aligned}
& P\left(\mathbf{s}^{k} \rightarrow \mathbf{c}^{k}\right) \leq \prod_{n=1}^{N}\left[\frac{1}{1+\frac{P_{t} \sigma_{n}^{2}}{4 N_{0} \varepsilon_{n}}\left|\mathbf{a}_{n}^{\mathrm{T}} \Delta \mathbf{s}\right|^{2}} \times\right. \\
& \left.\left(1-\sum_{m=1, m \neq n}^{N} p_{m n}\right)+\sum_{l=1, l \neq n}^{N} p_{l n}+\text { H.O.T. }\right],
\end{aligned}
$$

where H.O.T. stands for the higher order terms of the SERs. When SNR is high, the SERs are small and the higher order terms can be ignored. Specific derivation is omitted due to space limitations.

Diversity is an important criterion since it determines the slop of the performance curve. However, it is obvious from (21) that $\left|\mathbf{a}_{n}^{\mathrm{T}} \Delta \mathbf{s}\right|=0$ would result in the loss of diversity. In order to achieve the maximum diversity, the first criterion of the network coding vectors is derived as follows

Diversity criterion: The full diversity gain $N$ can be achieved if the following maximum diversity condition holds true for any distinct pair $\left\{\mathbf{s}^{k}, \mathbf{c}^{k}\right\}$,

$$
\left|\mathbf{a}_{n}^{\mathrm{T}} \Delta \mathbf{s}\right| \neq 0, \quad \forall n \in[1, N], \forall \mathbf{s}^{k}, \mathbf{c}^{k} \in \mathcal{A}^{N} .
$$

Theorem 1: Let $d_{\max }$ represent the maximum Euclidean distance of $\mathcal{A}$. If $\forall n \in[1, N],\left|\mathbf{a}_{n}^{\mathrm{T}} \Delta \mathbf{s}\right| \neq 0$ for any distinct pair $\left\{\mathbf{s}^{k}, \mathbf{c}^{k}\right\}$, the PEP under high SNR is upper bounded by

$$
P\left(\mathbf{s}^{k} \rightarrow \mathbf{c}^{k}\right)<\left(G \frac{P_{t}}{N_{0}}\right)^{-N},
$$

where

$G=\left[\prod_{n=1}^{N} \frac{1}{\left|\mathbf{a}_{n}^{\mathrm{T}} \Delta \mathbf{s}\right|^{2}}\left(\frac{4 \varepsilon_{n}}{\sigma_{n}^{2}}+\sum_{l=1, l \neq n}^{N} \frac{N C_{M} \xi_{l} d_{\max }^{2}}{\sigma_{l n}^{2}}\right)\right]^{-\frac{1}{N}}$

Proof is omitted due to space limitations.

It can be seen from Theorem 1 that given signal constellation and channel condition, the PEP depends on the design of network coding vectors. To get a better performance, the second design criterion is derived as follows.

Product criterion: The minimum value of the product $\prod_{n=1}^{N}\left|\mathbf{a}_{n}^{\mathrm{T}} \Delta \mathbf{s}\right|^{2}$ over all pairs of distinct signal vectors $\mathbf{s}^{k}$ and $\mathbf{c}^{k}$ should be as large as possible.

The product criterion is of secondary importance and should be optimized if full diversity is achieved.

\section{B. Design of DSTNC}

Let $\mathbf{A}_{N}$ denote the combining matrix (network coding matrix), which is constructed from the network coding vectors of the $N$ user nodes as follows

$$
\mathbf{A}_{N}^{\mathrm{T}}=\left[\begin{array}{lllll}
\mathbf{a}_{1} & \cdots & \mathbf{a}_{n} & \cdots & \mathbf{a}_{N}
\end{array}\right] .
$$

Let $\mathbf{s}_{f}=\mathbf{A}_{N} \mathbf{s}$, where $\mathbf{s}=\left[s_{1}, \ldots, s_{N}\right]^{\mathrm{T}} \in \mathcal{A}^{N}$. According to the diversity criterion, $\mathbf{A}_{N}$ should be designed to satisfy the following property: the original signal constellation is rotated and expanded such that there is no zero entry in the vector $\mathbf{s}_{f}$ for any $\mathbf{s} \neq \mathbf{0}_{N}$. This is referred to as the signal space diversity technique [13] [14]. According to the product criterion, the

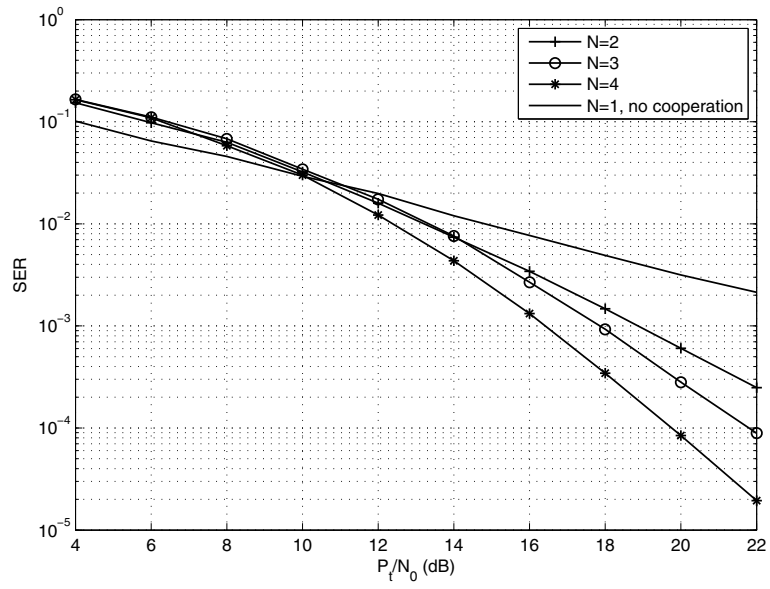

Fig. 2. SER performance for different number of user nodes with $d_{u}=d / 3$

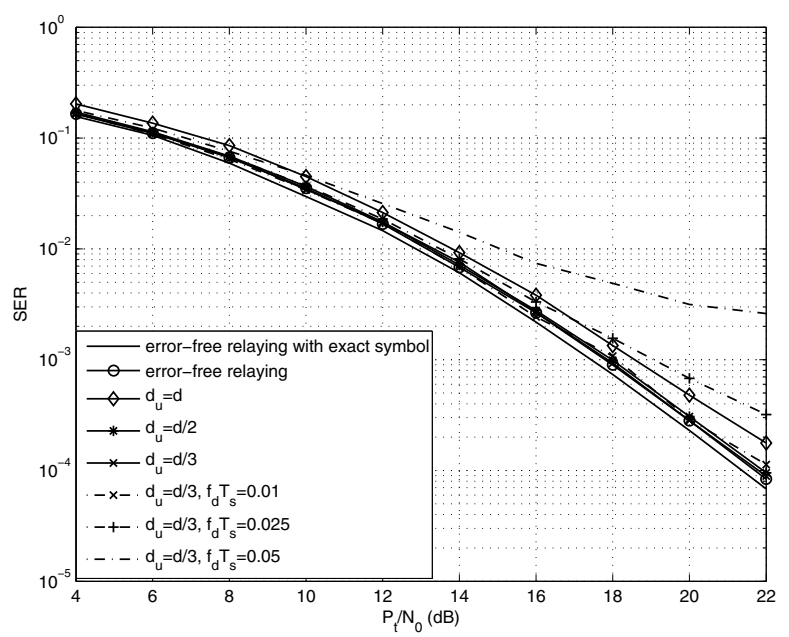

Fig. 3. SER performance for different distances and Doppler frequencies with $N=3$

minimum product distance of a set of signal points should be maximized, which is investigated in [13], [14], and [15]. For any number of $N$, the matrix $\mathbf{A}_{N}$ can be constructed as

$$
\mathbf{A}_{N}^{\mathrm{T}}=\frac{1}{\sqrt{N}}\left[\begin{array}{cccc}
1 & 1 & \cdots & 1 \\
\theta_{1} & \theta_{2} & \cdots & \theta_{N} \\
\vdots & \vdots & \ddots & \vdots \\
\theta_{1}^{N-1} & \theta_{2}^{N-1} & \cdots & \theta_{N}^{N-1}
\end{array}\right]
$$

and the network coding vector for $U_{n}$ is

$$
\mathbf{a}_{n}=\left[\begin{array}{llll}
1 & \theta_{n} & \cdots & \theta_{n}^{N-1}
\end{array}\right]^{\mathrm{T}},
$$

where $\theta_{n}$ is determined by the number of nodes and the construction method. More details can be found in [13]-[15].

\section{Simulation Results}

In the simulations, the user nodes have the same distance from $D$, denoted by $d$. The channel variance between the 
user nodes to $D$ is assumed to be 1 . The inter-user distances are the same and denoted by $d_{u}, d_{u} \leq d$. The path loss exponent is $\alpha=3$. The channel coefficients follow the Jakes' model [16] with Doppler frequency $f_{d}$ and normalized fading parameter $f_{d} T_{s}$, where $T_{s}$ is the sampling period which equals the symbol period. Unless specified otherwise, $f_{d} T_{s}=0.0025$ is used throughout the simulation. The power constraint of each user is $P_{t}$. Various numbers of user nodes are used with $N=2,3$, and 4 . For $N=2,4, \theta_{n}=e^{\frac{j n-3}{2 N} \pi}$, and for $N=3$, $\theta_{n}=e^{j\left(\frac{1}{9}+\frac{2(n-1)}{3}\right) \pi}$ with $n \in[1, N]$. The corresponding network coding vectors are generated according to (27).

Fig. 2 shows symbol error rate (SER) performance versus SNR $\frac{P_{t}}{N_{0}}$ of the DSTNC scheme with $N=2,3,4$ user nodes. $d_{u}=d / 3$ and BPSK is adopted in the simulation. For a comparison, the single node scenario without cooperation is also considered. To make a fair comparison, the user node transmits each symbol twice. Since the channel does not change in two adjacent time slots, time diversity is not available for the single node scenario. It can be seen from Fig. 2 that, the performance of single node without cooperation is always poor with the SER above $10^{-3}$ for $P_{t} / N_{0} \leq 22 \mathrm{~dB}$. In moderate and high SNR regimes, the SER performance is improved by user cooperation obviously, and the improvement becomes more significant as the number of cooperation nodes increases, due to the increase in diversity order. From the curves, we verify that the full diversity order, which is equal to the number of user nodes $N$, is achieved by the proposed DSTNC scheme. When SNR is low, the cooperative transmission shows no performance advantage. This is because the user nodes do not relay most of the received symbols due to the high SER.

Fig. 3 depicts the SER performances for different inter-user distances with $N=3$. It can be seen that as the inter-user distance $d_{u}$ decreases, the SER performance becomes better. When $d_{u}=d / 3$, the performance of the proposed scheme with BPSK modulation is almost the same as that of the errorfree relaying case, which is similar to a MIMO system with $N$ transmit antennas and single receive antenna. Compared to the error-free relaying case, the performance degradation for $d_{u}=d / 2$ is also neglectable, while a performance gap exists when $d_{u}=d$. These observations match the analysis in Section III. From Theorem 1 we can see that the PEP is influenced by the inter-user distance through $\sigma_{l n}^{2} \propto d_{l n}^{-\alpha}$. In many practical situations with adjacent cooperative nodes, the proposed DSTNC scheme can obtain a performance similar to the MIMO system while overcoming some practical issues in cooperative communications. In the differential detection, the signal terms in the equivalent noise variances of (7) and (10) are replaced by their mean values. To show the effect of this substitution, the SER performance of the ideal case using exact symbols in detection is also plotted in Fig. 3. From the solid and circled curves we can see that, compared to the ideal case, the SER performance with mean value is slightly worse.

SER performance for different Doppler frequencies are also presented in Fig. 3. Because the differential scheme requires the channels do not change significantly during a period of two adjacent transmissions, the performance degrades as the Doppler frequency increases, associated with decreasing channel coherence time. We can see from Fig. 3 that the performance degradation is quite small when $f_{d} T_{s}$ increases to 0.01 , but error floors appear when $f_{d} T_{s} \geq 0.025$. Although the performance of DSTNC degrades when the channel changes rapidly, it still can work in some situations where coherent detection is impractical.

\section{CONCLUSION}

In this paper, we consider the practical challenges in timing and frequency synchronizations and channel estimation for multinode cooperative communications and propose a new differential transmission scheme called DSTNC to overcome such issues. The proposed scheme utilizes linear network coding to combine the information and reduce the required time slot. The PEP performance at the destination node is analyzed. Design criteria of the network coding vectors are derived. Compared with the traditional multinode cooperative communication using TDMA, the proposed DSTNC can achieve full diversity with a significant reduction in transmission delay.

\section{REFERENCES}

[1] J. N. Laneman, D. N. C. Tse, and G. W. Wornell, "Cooperative diversity in wireless networks: efficient protocols and outage behavior," IEEE Trans. Inform. Theory, vol. 50, no. 12, pp. 3062-3080, Dec. 2004.

[2] Y. Li and X. G. Xia, "A family of distributed space-time trellis codes with asynchronous cooperative diversity," IEEE Trans. Commun., vol. 55, no. 4, pp. 790-800, Apr. 2007

[3] Q. Zhao and H. Li, "Differential modulation for cooperative wireless systems," IEEE Trans. Signal Process., vol. 55, no. 5, pp. 2273-2283, May 2007.

[4] G. Wang, Y. Zhang, and M. Amin, "Differential distributed space-time modulation for cooperative networks," IEEE Trans. Wireless Commun. vol. 5, no. 11, pp. 3097-3108, Nov. 2006.

[5] Y. Shang and X. G. Xia, "Shift-full-rank matrices and applications in space-time trellis codes for relay networks with asynchronous cooperative diversity," IEEE Trans, Inform. Theory, vol. 52, no. 7, pp. 3153-3167, Jul. 2006.

[6] M. O. Damen and A. R Hammons, "Delay-tolerant distributed TAST codes for cooperative diversity," IEEE Trans. Inform. Theory, vol. 53, no. 10 , pp. $3755-3773$, Oct. 2007.

[7] H. Q. Lai, A. Ibrahim, and K. J. R. Liu, "Wireless network cast: Locationaware cooperative communications with linear network coding," IEEE Trans. Wireless Commun., vol. 8, no. 7, pp. 3844-3854, Jul. 2009.

[8] H. Q. Lai and K. J. R. Liu, "Multipoint-to-point and point-to-multipoint space-time network coding," in Proc. IEEE ICASSP, Dallas, March 2010.

[9] E. Fasolo, F. Rossetto, and M. Zorzi, "Network coding meets MIMO," in Proc. Workshop Network Coding, Theory, Appl., Hong Kong, Jan. 2008, pp. 1-6.

[10] A. K. Sadek, W. Su, and K. J. R. Liu, "Multinode cooperative communications in wireless networks," IEEE Trans. Signal Process. vol 55, no. 1, pp. 341-355, Jan. 2007

[11] V. Tarokh, H. Jafarkhani, and A. R. Calderbank, "Spcae-time block codes from orthogonal design," IEEE Trans. Inform. Theory, vol. 45, no.5, pp. 1456-1467, Jul. 1999.

[12] K. J. R. Liu, A. K. Sadek, W. Su, and A. Kwasinski, Cooperative Communications and Networking, Cambridge University Press, 2008.

[13] X. Giraud, E. Boutillon, and J. C. Belfiore, "Algebraic tools to build modulation schemes for fading channels," IEEE Trans. Inform. Theory, vol. 43, no. 3, pp. 938-952, May 1997.

[14] J. Boutros and E. Viterbo, "Signal space diversity: a power and bandwith efficient diversity technique for the Rayleigh fading channel," IEEE Trans. Inform. Theory, vol. 44, no. 4, pp. 1453-1467, Jul. 1998.

[15] Y. Xin, Z. Wang, and G. B. Giannakis, "Space-time diversity systems based on linear constellation precoding," IEEE Trans. Wireless Commun., vol. 2, no. 2, pp. 294-309, Mar. 2003.

[16] W. C. Jakes, Microwave Mobile Communications, Piscataway, NJ: IEEE Press, 1993. 